\section{Warum das Thema „TA und Industrie" umfunktionieren in eine Generaldiskussion über TA? - Eine Erwiderung auf Hauke Fürste nwerth}

\author{
von Armin Grunwald, ITAS
}

\section{Vorbemerkung}

Einladungen $\mathrm{zu}$ einer schriftlichen Diskussionsrunde sind nicht ohne Risiko. Zwar wird oft beklagt, dass zuviel monologisiert werde, ohne dass Austausch und Dialog stattfinden. Dies hört man gelegentlich sogar in der doch recht kleinen TA-Community, wo man sich gegenseitig kennt. Aber: Austausch und Dialog machen Arbeit, jedenfalls wenn sie in schriftlicher Form erfolgen sollen. So war ich selber neugierig, ob die Einladung im Heft 3/2000 der TADatenbank-Nachrichten überhaupt irgendeine Resonanz haben würde über die gelegentlichen lobenden oder kritischen Bemerkungen der Leser hinaus.

Das Experiment scheint gelungen. Bereits im Heft 4/2000 konnten die Beiträge von Utz Schäffer und Hauke Fürstenwerth abgedruckt werden. Der Beitrag von Axel Zweck war schon damals eingereicht, fiel aber einer technischen Panne zum Opfer und wird daher im aktuellen Heft publiziert, neben dem Beitrag von Hans-Jochen Luhmann. Allen Mitdiskutierern ein ganz herzliches Danke! Es gibt bereits eine ganze Reihe positiver Rückmeldungen aus dem Leserkreis der TA-Datenbank-Nachrichten zu dieser Diskussion, vor allem dazu, dass sie erstens überhaupt in dieser Öffentlichkeit stattfindet, und dass sie zweitens nicht nur öffentlich, sondern auch offen geführt wird. Zur Dokumentation der verschiedenen Positionen und Argumente wird das Heft 2/2001 dem Schwerpunkt „TA und Wirtschaft" gewidmet sein.

Als derjenige, der diese Diskussionsrunde angestoßen hat, kann es nicht meine Aufgabe sein, in der noch laufenden Diskussion zu den einzelnen Diskussionsbeiträgen kritisch Stellung zu beziehen. Im Folgenden sehe ich mich dennoch veranlasst, auf den Beitrag von Hauke Fürstenwerth einzugehen, weil dieser einerseits erheblich über eine Positionsbestimmung zum
Thema „TA und Wirtschaft“" hinausgeht und ganz allgemeine Thesen zur TA äußert, und weil ich andererseits diesbezüglich an mehreren Punkten direkt angesprochen wurde.

\section{Exkurs: Generaldiskussion über TA}

Hauke Fürstenwerth nutzt das Thema „TA und Industrie" für eine Generalabrechnung mit der TA: „Institutionell betriebene TA hat versagt“ scheint die Hauptmotivation zu sein, ganz auf der Linie der WHU-Studie. Ich bin etwas überrascht über diese Wendung, werden darin doch alte ideologische Gräben wieder sichtbar, die als überwunden galten: hier die Industrie, die „eigenverantwortlich“ Technik produziert, dort die TA, die ,erbitterten ideologischen Streit" führt, ohne greifbare Ergebnisse zu produzieren. Öffentlich geförderte TA sei überflüssig, weil sie nur mit sich selbst befasst sei und dadurch eine mögliche Nachfrage der Industrie wo ja schließlich Technikentwicklung stattfindet - selbst verbaut.

Ich möchte an dieser Stelle die Diskussion nicht auf diese allgemeine Ebene ausdehnen, denn dort ist nicht viel Gutes zu erwarten. Verpflichtet fühle ich mich allerdings, in aller Kürze, zu einigen Klarstellungen bzw. zum Widerspruch an einigen Stellen. Ich habe nach der Lektüre des Beitrages von Hauke Fürstenwerth meinen eigenen „Aufreißer“ der Diskussion (Heft 3/2000) noch einmal durchgeschaut. Die Attribute „emotionsgeladen“ (S. 161) und „fundamentalistisch“ (S. 163) kann ich dem Text beim besten Willen nicht zuerkennen. Die Unterscheidung in der Überschrift „Politikberatung oder Unternehmensberatung " mag man zwar mit Recht als rhetorisch überzogen und zu pauschal bezeichnen - das ist eben auch die textstrategische Funktion einer Überschrift, die Leser interessieren und zum Diskutieren anregen soll -, aber wohl kaum als fundamentalistisch. Anders ausgedrückt: ein ,oder“ muss ja nicht ein ausschließendes „oder“ sein. Warum ein Fundamentalismus-Vorwurf, wenn es darum geht, zwei Typen von Beratung (der Wirtschaft bzw. der Politik) mit ihren je verschiedenen Zwecken und Randbedingungen zu unterscheiden?

Der tiefere Anlass für den Beitrag scheint sich im Teil 2 zu finden (S. 158ff.). Dort wird ein Gesellschaftsmodell entwickelt, nach dem 
die Wirtschaft, von den Zwängen der Obrigkeit befreit, autonom und eigenverantwortlich über Technik entscheidet, und dies wird als Quelle unseres Wohlstandes angesehen. ${ }^{1}$ Das hat sicher in gewissen Aspekten seine Berechtigung, und es gibt mit Grund wohl kaum noch jemanden, der meint, der Staat solle die Technikentwicklung zentral steuern. Aber in anderen Hinsichten kann man dieses Modell als zynisch interpretieren: vielleicht hat es wirklich einmal jemanden gegeben, der ,klare Visionen ... über Nutzen und Vorteile" der Tiermehlverfütterung hatte und der ,,an deren Realisierung mit großer Verbissenheit gearbeitet" hat. Ob BSE wirklich etwas mit Tiermehl zu tun hat, sei dahingestellt. Aber die zentrale Frage ist doch, reicht es, wenn ein Technikentwickler „klare Visionen ... über Nutzen und Vorteile“ einer Technik hat und, an deren Realisierung mit großer Verbissenheit" arbeitet, um anzunehmen, dass diese Technik das gesellschaftliche Wohlbefinden steigert? Ich glaube, hier gibt es genügend Gegenbeispiele. Und diese rechtfertigen es, eben nicht die Wirtschaft als autonomen Bereich anzusehen, sondern bestimmten Rahmenbedingungen $\mathrm{zu}$ verpflichten (wie zur Zeit durch die Einrichtung eines Ministeriums für Verbraucherschutz wieder stärker im öffentlichen Bewusstsein). TA hat es - zumindest auch - mit der Gestaltung dieser Rahmenbedingungen zu tun, und diese Gestaltung ist nun einmal mit guten Gründen nicht in das Belieben der Wirtschaft gestellt. ${ }^{2}$ Liegt hier der eigentliche Kern des Dissenses? Dann wäre es ein Dissens in gesellschaftstheoretischer Hinsicht.

Die kritische Bemerkung von Hauke Fürstenwerth zu meinem Buch über ,Rationale Technikfolgenbeurteilung (S. 161 und Anmerkung 10) beachtet nicht, dass dieses Buch ganz explizit keine umfassende Theorie der TA zu geben beansprucht, sondern dass es den Möglichkeiten wissenschaftlicher Erkenntnis in diesem Bereich gewidmet ist und darum ganz zwangsläufig eine stark wissenschaftstheoretische Schlagseite hat.

Sodann möchte ich auf zwei „nette“ und dann doch etwas versöhnlich stimmende Selbstwidersprüche hinweisen: erstens zitiert Hauke Fürstenwerth am Anfang Thomas Petermann, der vor übertriebenen Erwartungen an TA warnt, und nimmt dies als Beleg, dass TA nichts zu bieten habe. In Teil 3 warnt er dann aber vor „unverschämten“ Ansprüchen der TA und ruft zur Bescheidenheit auf. Was denn nun?

Zweitens, nach der Prognose, dass es bald keine TA-Institutionen mehr geben wird, schreibt Hauke Fürstenwerth, dass die Auftraggeber von TA ,bei Bedarf entsprechende Studien bei einschlägig qualifizierten Forschungseinrichtungen und Beratungsunternehmen in Auftrag geben ....". Da sind sie ja wieder, die TA-Einrichtungen. Bedarf gibt es genug, wie unsere Auftragsbücher zeigen, und es gibt $\mathbf{h}$ stitutionen, die den Bedarf abdecken.

\section{Zurück zum Thema}

Zum Thema selbst hat Hauke Fürstenwerth eine Reihe weiterführender Fragen gestellt (Teil 1 seines Beitrages). Die Stichworte Cemeinwohl, Partizipation, Langfristigkeit von Technikentscheidungen in der Wirtschaft, die Diskussion um Rahmenbedingungen für die Technikentwicklung sind sicher zentrale Aspekte. Auch über die Art der Fragestellungen kann man reden, auch darüber, ob sie der Praxis in der Wirtschaft angemessen sind oder nicht (wobei dies aber wohl nicht die einzige Perspektive ist, unter der man die Wahl der Fragestellungen beurteilen sollte). Auch dazu soll die Diskussionsrunde dienen, und dazu sollte man sich auch gelegentlich zusammensetzen und weiterdiskutieren. Es zeigt sich aber deutlich, dass eine Diskussion über TA nie nur eine Diskussion über TA ist, sondern dass allgemeine Fragen der Technikpolitik, Unterschiede im Wirtschaftsverständnis, Überlegungen zum Verhältnis von Wissenschaft und Politik etc. hineinspielen - genau das macht TA gleichermaßen interessant wie auch schwierig.

\section{Anmerkungen}

1) Die Argumentation, dass Technikentwicklung in der globalisierten Ökonomie genau so verlaufe, und dass deswegen TA dort erfolgen soll, wo diese stattfindet, wird auch in Teilen der TA geteilt (Steinmüller et al. 1999).

2) Vielem in diesem Teil kann ich durchaus zustimmen, sogar so vielem, dass mir manchmal nicht mehr ganz klar ist, warum Hauke Fürstenwerth zu derart abweichenden Urteilen kommt. Der Grund scheint darin zu liegen, 
dass in seinem Gesellschaftsmodell weder Rahmenbedingungen für die Wirtschaft noch Nebenfolgen von Technik vorkommen. Damit ignoriert er wesentliche Ergebnisse der Technikforschung aus den letzten Jahrzehnten.

\section{Literatur}

Steinmüller, K.; Tacke, K.; Tschiedel, R., 1999: Innovationsorientierte Technikfolgenabschätzung. In: S. Bröchler; G. Simonis; K. Sundermann (Hg.): Handbuch Technikfolgenabschätzung, Band 1, Berlin, S. 129-145

\section{Kontakt}

Prof. Dr. Armin Grunwald

Forschungszentrum Karlsruhe $\mathrm{GmbH}$

Institut für Technikfolgenabschätzung

und Systemanalyse (ITAS)

Postfach 3640, D-76021 Karlsruhe

Tel.: + 49 (0) $7247 / 82-2500$

Fax: + 49 (0) 7247 / $82-4806$

E-Mail: grunwald@itas.fzk.de 